\title{
Novel Full-Band Waveguide Polar Modulator for Radio Astronomy Applications
}

\author{
Roger Hoyland, Juan L. Cano ${ }^{\circledR}$, Abdelwahed Tribak, Angel Mediavilla, and Eduardo Artal, Member, IEEE
}

\begin{abstract}
A novel full-band $(40 \%$ relative bandwidth $)$ waveguide polar modulator (WPM) design based on a new architecture with two turnstile junctions and a nearly frequencyindependent differential $180^{\circ}$ phase shift between orthogonal arms is presented. The use of suitably designed turnstile junctions together with antiphase waveguide-coaxial-waveguide transitions in reduced-height waveguide technology enables the improvement of bandwidth over previous WPMs with a very compact and simple solution. This design enables continuous rotation about its central axis at 40 revolutions/s, which makes the modulator suitable for receivers with significant $1 / f$ noise such as cryogenic radiometers. The polar modulator presented in this paper is designed to be cooled down to cryogenic temperatures in order to reduce its contribution to the receiver noise. This design is suitable for radio astronomy applications due to its costeffective, compact design, and high quality performance as well as being readily scalable. A polar modulator in the Ka-band (26.5-40 GHz) has been designed to show a reflection better than $-20 \mathrm{~dB}$, an insertion loss around $0.3 \mathrm{~dB}$, and a cross-coupling near $-35 \mathrm{~dB}$ measured at room temperature $(T=298 \mathrm{~K})$. This insertion loss is reduced by $\mathbf{3 0 \%}$, while the phase difference and cross-coupling maintain similar values when the polar modulator is cooled to approximately $T=80 \mathrm{~K}$.
\end{abstract}

Index Terms-Cryogenics, polarimeter, polarimetry, radiometry, turnstile junction.

\section{INTRODUCTION}

$\mathbf{R}$ OTATION of the incoming signal's polarization state is often required in many communications systems where the receiver is only sensitive to a determined linear polarization. Thus, antenna systems designed to receive this single polarization are able to work with different polarizations increasing their versatility. Traditionally, this rotation has been carried out by cascading wire grids rotated at different angles producing fixed input-output rotation angles [1], [2] or using alternative structures based on meander lines resulting in arbitrary rotation angles [3]. In addition to communication

Manuscript received November 10, 2017; revised February 5, 2018 and March 21, 2018; accepted March 30, 2018. This work was supported by the Spanish Ministry of Economy and Competitiveness under the CONSOLIDERINGENIO 2010 program and project CSD2010-00064. (Corresponding author: Juan L. Cano.)

R. Hoyland is with the Instituto de Astrofísica de Canarias, 38660 Santa Cruz de Tenerife, Spain (e-mail: rjh@iac.es).

J. L. Cano, A. Mediavilla, and E. Artal are with the Departamento de Ingeniería de Comunicaciones, Universidad de Cantabria, 39005 Santander, Spain (e-mail: juanluis.cano@unican.es; angel.mediavilla@unican.es; eduardo.artal@unican.es).

A. Tribak is with the Institut National des Postes et Telecommunications, Rabat, Morocco (e-mail: tribak@inpt.ac.ma).

Color versions of one or more of the figures in this paper are available online at http://ieeexplore.iee.org.

Digital Object Identifier 10.1109/TMTT.2018.2829177 applications, radio astronomy and, in particular, the study of the polarization component of the cosmic microwave background (CMB), has emerged as a new area demanding high-performance polarization rotators or polarizers which frequently need to operate in continuous rotation in order to modulate this incoming linear component so as to become measurable over the unpolarized background. Due to the importance of this polarized signal for cosmology, which is some orders of magnitude weaker than the $\mathrm{CMB}$ power spectrum, a variety of experiments are running or are planned worldwide to measure the CMB polarization [4]. For these experiments, very sensitive and high-quality receivers are needed, where the minimization of the cross-polarization component while maintaining a large focal plane array is of utmost importance to maximize the sensitivity.

As a key component in microwave polarizers, the polar modulation device defines to a great extent the performance of the receiver itself. When continuous rotation is required, these devices have been usually designed based on the socalled half-wave plate (HWP) with its various configurations. HWPs are quasi-optical polarization modulators that shift the phase $\left(180^{\circ}\right.$ phase shift) between the orthogonal components of the incoming radiation. The HWP is rotated along its central axis producing the polar modulation effect that enables the cancelation of systematic errors in the receiver. The speed of rotation is directly related to the level of $1 / f$ noise that can be overcome. These HWPs are placed before the horns in the instrument so they are the first optical element, avoiding the modulation induced by other subsystems. Moreover, they are generally cooled to cryogenic temperatures to minimize their contribution to the system noise temperature and thus maximizing the instrument sensitivity.

The different technologies of HWPs can be divided into two main categories: reflective and transmissive modulators. The former use a wire grid located at a specific distance from a mirror which presents narrowband performance [5]-[7] although recent developments have obtained multioctave bandwidths [8]. In the latter, the radiation goes through a rotating thin birefringent plate producing the phase difference, which is related with the plate thickness [9]. Evolved designs have increased the bandwidth [10]-[12]. A good summary of HWPs technologies and performances can be found in [13].

The main drawback these HWPs face is that they are complex structures with ultimately limited diameters. Therefore, the technology constrains the focal plane size and, as a consequence, the maximum number of receivers that can be allocated and/or their use in frequency bands below W-band. 
Furthermore, the rotation speed is a serious limitation for these structures, which has an impact on the systematic calibration and $1 / f$ suppression.

As an alternative to HWPs, a few waveguide polar modulators (WPMs) have been developed. These polarizers are placed between the feed horn and the orthomode transducer (OMT) in the receiver chain; hence, every receiver in the instrument requires its own polarizer (in the case of multipixel cameras for example). The advantages of this strategy regarding HWPs are smaller polarizers that can be spun faster, easily scalable instruments, easier cryogenic cooling, and optics cross-polarization effectively defined by the feed horn rather than the polarizer. Obviously, the receiver performance would benefit from a whole system design taking into account all the subsystems. An example of WPMs is the Faraday rotator [5]. This polarizer uses superconducting solenoids wound around the waveguide to produce the phase shift in the incoming radiation for bandwidths of $30 \%$. However, the necessity of the superconducting technology may represent an additional drawback. A different approach is the adaptation of the principles in [10] to a waveguide structure [14]. This solution provides very good electrical performance at millimeter-wave frequency ranges with a $30 \%$ bandwidth. WPMs are a good solution for single pixel up to medium sized pixel arrays and experiments working at frequencies below W-band. For a large number of pixels in the millimeter frequency range a single HWP solution is more practical.

In this paper, a waveguide polar modulator is designed using a novel implementation based on two turnstile junctions connected by $180^{\circ}$ differential branches accomplished with antiphase electric field coupling. This solution, implemented completely in waveguide technology, relies on the electric field direction change between branches to obtain the desired phase difference, therefore enabling a full-band performance (40\%) without dielectric layers or complicated arrangements. Moreover, the simplicity of its mechanical concept permits a compact design that can be easily scaled, repeated, and cryogenically cooled, which makes it very suitable for modern radio astronomy experiments with multipixel instruments. The proposed polar modulator was designed for the first instrument of the QUIJOTE CMB experiment [15].

An overview of the QUIJOTE first instrument principle of operation is depicted in Section II. The electrical design and simulation results of the polar modulator are presented in Section III. Section IV provides details of the mechanical implementation, whereas characterization procedure and experimental results, both at room and cryogenic temperatures, are shown in Section V. Finally, conclusions are given in Section VI.

\section{QUIJOTE FIRST INSTRUMENT PRINCIPLE OF OPERATION}

The receiver scheme in the first instrument of the QUIJOTE experiment is presented in Fig. 1. The cosmic microwave radiation enters the receiver chain through a corrugated feed horn with high gain, low reflection, and minimum crosspolarization. After the feed horn, the polar modulator is

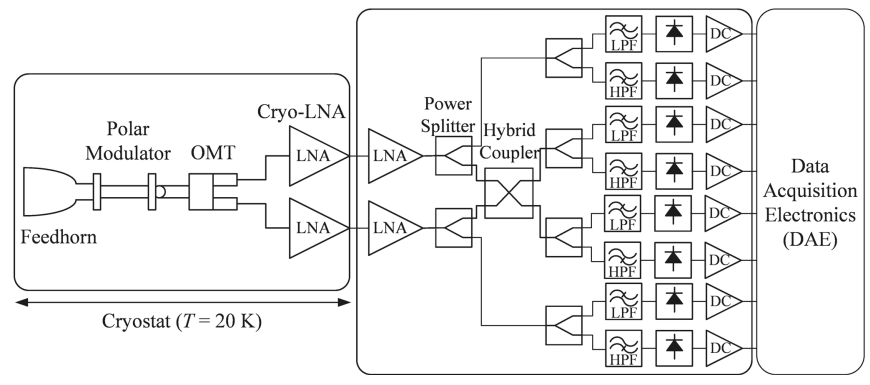

Fig. 1. Receiver scheme for each pixel in the first instrument of the QUIJOTE experiment.

placed and rotated continuously with a physical frequency of $10-40 \mathrm{~Hz}$ (giving rise to a signal modulation of 40-160 Hz). The polar modulator output is fed into an OMT [16] which provides the two orthogonal polarizations that are later amplified in two cryogenically cooled low-noise amplifiers. Then, these signals pass through a room temperature module where they are further amplified, separated in successive power splitters, and spectrally filtered, in such a way that the initial bandwidth is separated into four lower-sideband channels and another four upper-sideband channels, each with half bandwidth of the original band. A pair of these channels corresponds to the two orthogonal polarizations in the instrument original reference system ( $Q$ aligned) and the other gives two orthogonal polarizations expressed in a system rotated $45^{\circ}$ with respect to the previous one ( $U$ aligned). Therefore, (1) describing a linear polarimeter applied to our receiver scheme [17] and obtained after cascading the Jones matrices of each subsystem, gives the output of a wideband square-law detector proportional to the microwave power incident on it, in one subband, at a time $i$ as a function of the position angle of the modulator $\varphi_{i}$, regarding the instrument reference system, and the Stokes parameters $I_{i}, Q_{i}$, and $U_{i}$ (no circular polarization is assumed). Similar equations can be found for alternative receiver configurations [18]

$V_{i j}=\frac{1}{2} I_{i j}+\frac{1}{2} Q_{i j} \cos \left(4 \varphi_{i}+\phi_{j}\right)+\frac{1}{2} U_{i j} \sin \left(4 \varphi_{i}+\phi_{j}\right)$

where the index $j$ is $j=1,2,3$, and 4 for each of the four channels and the value of the phase $\phi_{j}$ is $0, \pi, \pi / 2$, and $-\pi / 2$, respectively. The polar modulator modulates the polarization of the incoming signal at a rate four times the modulator frequency, thereby defining the polar cycle. The integration of the signal over each polar cycle produces independent measurements of the incoming signal Stokes parameters. The possibility of a continuously spinning modulator gives a further defense against systematic errors since the signals are being constantly swapped between each of the four outputs. This modulation can completely null $1 / f$ noise which is generally common to each of the outputs and generated in the amplification elements in each chain. The nature of the $1 / f$ noise is that it is the dominant noise over longer time scales. There is a timescale where the $1 / f$ noise is no larger than the random white noise seen at the output of a radiometer. This frequency is known as the knee frequency of the radiometer. The modulator must completely switch the four outputs at a 


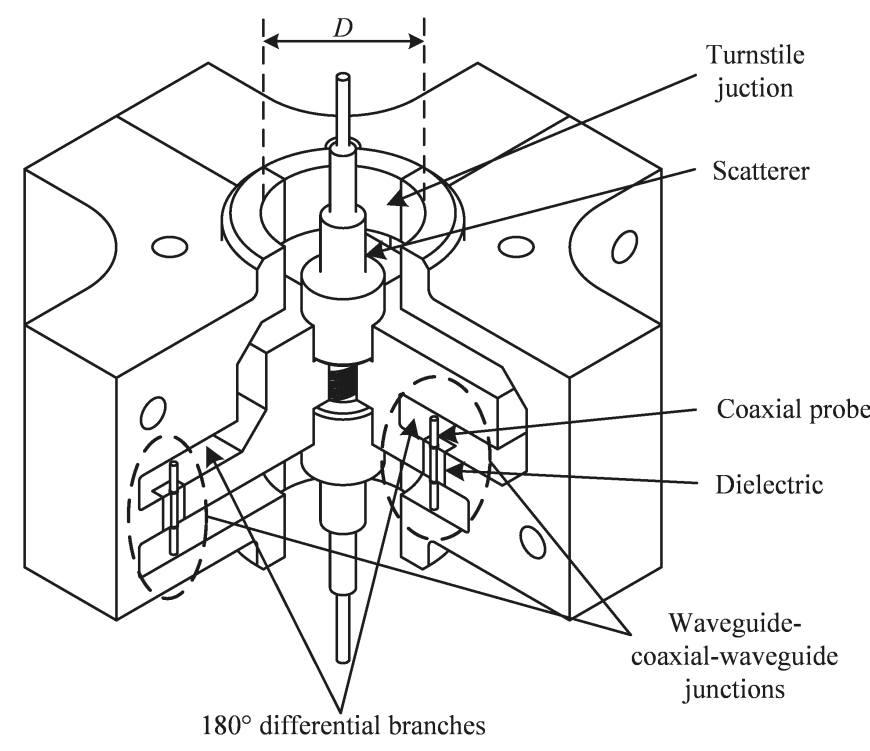

Fig. 2. Internal view of the polar modulator showing coaxial probes and turnstile junctions. The $180^{\circ}$ phase difference between branches is achieved through the coupling of the electric field in the opposite directions.

frequency higher than this knee in order to completely enable $1 / f$ removal from the data leaving only the random white noise and the polar signal. Measured data and a general description of the QUIJOTE first instrument can be found in [19].

\section{Polar Modulator Design and Simulation}

The first instrument of the QUIJOTE project aimed at three different bands: 10-14; 16-20; and 26-36 GHz; thus, three modulators were designed with wider bandwidths. All these modulators are scaled versions; therefore, only the design process and results of the exemplary in the Ka-band are presented, finding similar results but with reduced insertion loss in the lower bands.

A modulator can be made in waveguide by making use of a turnstile junction which has almost ideal properties [16], [20]. The polar modulator presented here is based on the turnstile junction design developed in [16] with input circular waveguide diameter $D=7.1 \mathrm{~mm}$ and reducedheight rectangular waveguide ports $(a=7.1 \mathrm{~mm}$ and $b=1.78 \mathrm{~mm}$ ). An incoming signal is split equally into four rectangular waveguides by the turnstile junction. Each orthogonal waveguide pair is sent through a path with $180^{\circ}$ difference to the other. A broadband $180^{\circ}$ path difference is achieved through a carefully thought out waveguide routing using equal and opposite $E$-plane bends and simple waveguide-coaxialwaveguide transitions and resulting in a symmetrical and an asymmetrical configuration. Finally, the four waveguides are brought together again into a second turnstile junction similar to the first.

A sketch of the designed polar modulator with one quarter removed is shown in Fig. 2. The four-stage scatterer of the two turnstile junctions can be seen to protrude out from the circular waveguide input and output. Two orthogonal reducedheight waveguide paths can be seen in either face of the

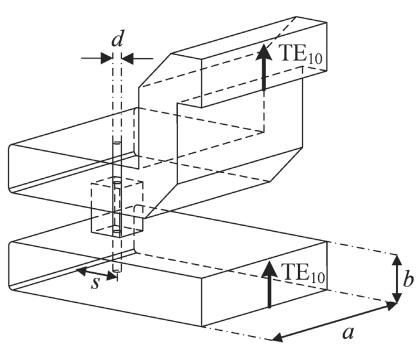

(a)

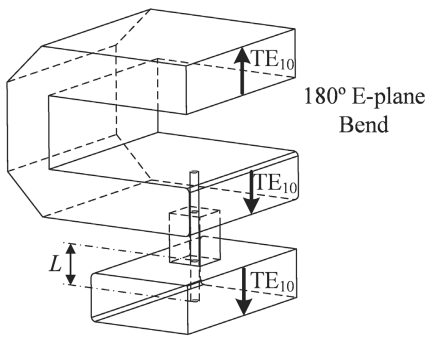

(b)
Fig. 3. Polar modulator branches. (a) With symmetric waveguide-waveguide connection. (b) With asymmetric waveguide-waveguide connection. Electrical length of both branches is identical.

cutaway. The polar modulator is conveniently made from four separate quadrants which are joined along the $E$-plane of each rectangular waveguide, which minimizes insertion losses. In the lower half of each face a center pin and dielectric can be seen adjoining top and bottom waveguide paths.

In Fig. 3, the two orthogonal branches are presented showing the relative phase of mode $\mathrm{TE}_{10}$ at given points in the rectangular waveguides. (The arrow indicates the electric field direction.) It is clear from the geometry of waveguide bends in the top waveguide section that antiphase signals result at the coaxial interfaces. The effect of the waveguidecoaxial-waveguide junction is to connect either side of a given rectangular waveguide to the same side of a second rectangular waveguide, thus the input and output interfaces are similar. In this design the number of waveguide bends is the same for both branches as well as the distance traveled through each.

The waveguide-to-waveguide transition is accomplished using $E$-plane antenna probes. A central conductor is designed to reach into both waveguides, whereas the dielectric is accommodated in the modulator structure. For the Ka-band, the central conductor is designed with diameter $d=0.31 \mathrm{~mm}$. The length and location of the probes are optimized for a broadband performance, in this case $L=1.45 \mathrm{~mm}$ long probes placed at $s=2.11 \mathrm{~mm}$ from the waveguide short circuit.

Simulation of both branches from Fig. 3 demonstrates the feasibility of the proposed structures to meet the electrical requirements over the entire frequency band. Fig. 4 shows the simulation results of the phase difference between both branches of the designed polar modulator. In Fig. 5, reflection results are presented showing a return loss better than $25 \mathrm{~dB}$. Insertion loss results are not presented due to their ideal values which are below $0.05 \mathrm{~dB}$.

The whole polar modulator structure in Fig. 6 was simulated using the frequency-domain solver in the CST Microwave Studio. Two different simulations were carried out. First, with $\varphi_{i}=0^{\circ}$ of polar angle between the polar modulator and the modes definition fixed by the OMT reference system, as shown in Fig. 6(a). With this simulation the reflection, transmission, cross-coupling, and phase difference between these two modes represent the results for $\varphi_{i}=0^{\circ}$ and $\varphi_{i}=90^{\circ}$ positions. And second, with $\varphi_{i}=45^{\circ}$ of polar angle, i.e., rotating the polar modulator structure $45^{\circ}$ while the excitation modes remain in the previous orientation defined by the OMT reference system, 


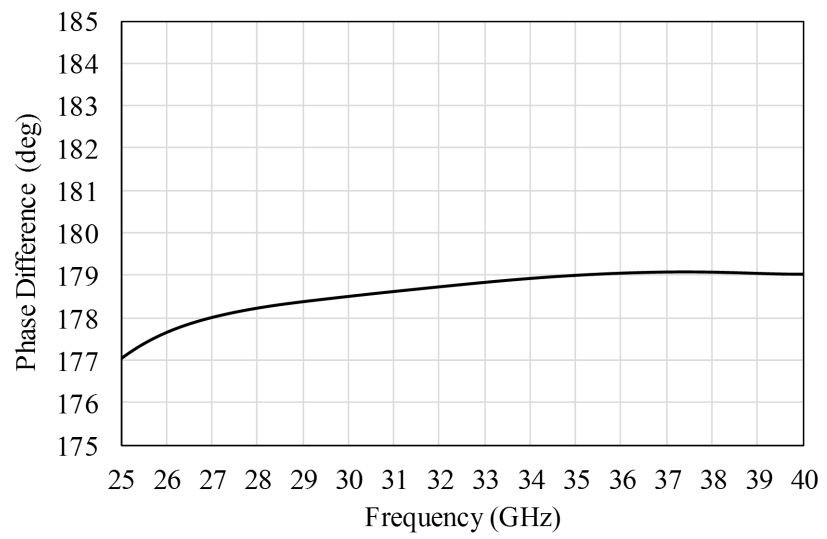

Fig. 4. Simulated phase difference between the polar modulator branches.

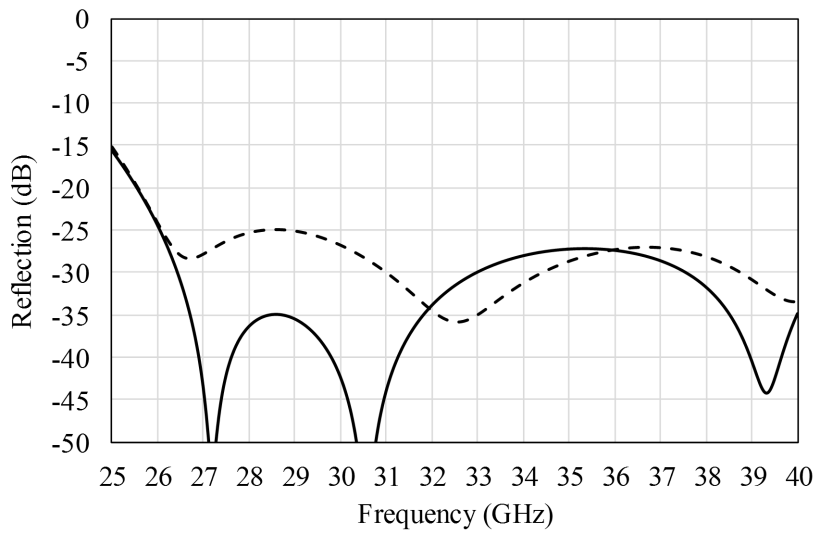

Fig. 5. Simulated reflection for both branches: symmetric waveguidewaveguide connection (solid line), asymmetric waveguide-waveguide connection (dashed line).

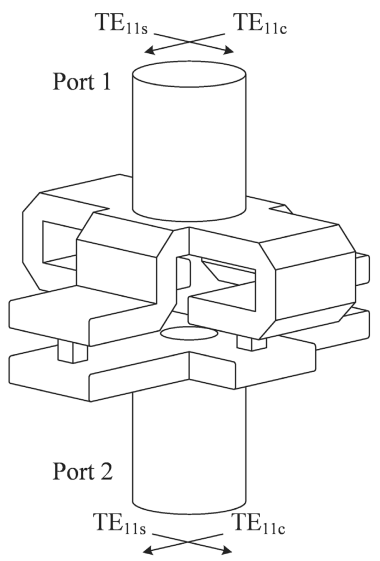

(a)

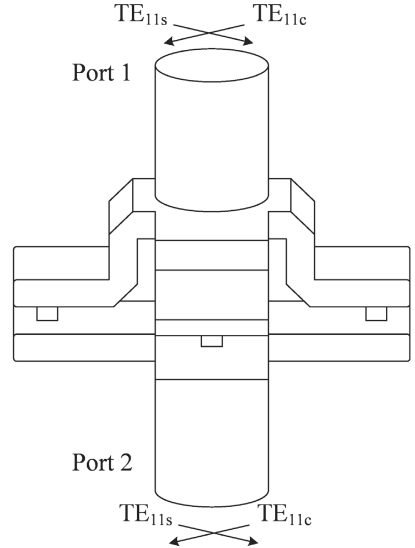

(b)
Fig. 6. Simulated structures. The orthogonal polarizations of mode $\mathrm{TE}_{11}$ are indicated in each port as $\mathrm{TE}_{11 \mathrm{~s}}$ and $\mathrm{TE}_{11 \mathrm{c}}$. (a) Polar modulator with $\varphi_{i}=0^{\circ}$ polar angle respect to the input port mode definition. (b) Polar modulator with $\varphi_{i}=45^{\circ}$ polar angle respect to the input port mode definition. The input port modes definition is defined by the OMT orientation, which remains fixed for both simulations.

as shown in Fig. 6(b). With this simulation, the reflection, transmission, cross-coupling and phase difference between these two modes represent the results for $\varphi_{i}=45^{\circ}$ and

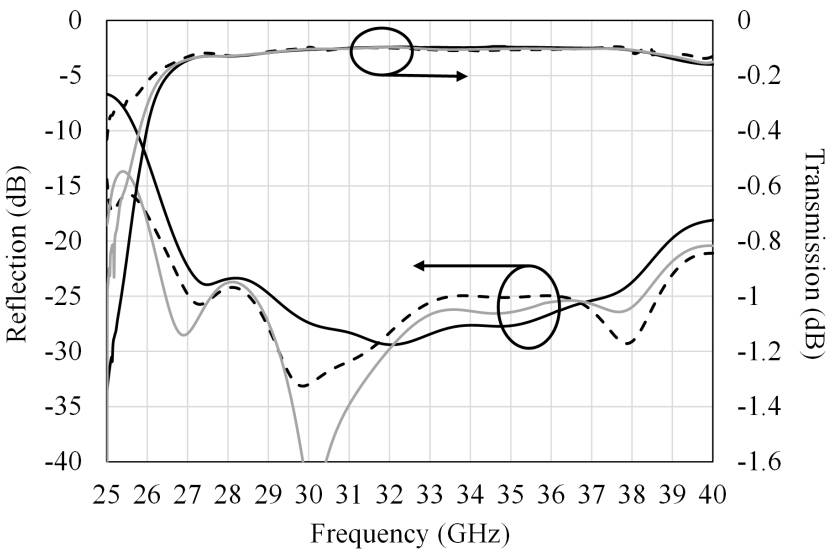

Fig. 7. Simulated reflection (left $y$-axis) and transmission (right $y$-axis) for the designed polar modulator: polar angle $\varphi_{i}=0^{\circ}$ [mode $\mathrm{TE}_{11 \mathrm{c}}$ in Fig. 6(a)] with black solid line, polar angle $\varphi_{i}=90^{\circ}$ [mode $\mathrm{TE}_{11 \mathrm{~s}}$ in Fig. 6(a)] with black dashed line, and polar angle $\varphi_{i}=45^{\circ}$ [mode $\mathrm{TE}_{11 \mathrm{c}}$ in Fig. 6(b)] with gray solid line. Results for $\varphi_{i}=135^{\circ}$ [mode $\mathrm{TE}_{11 \mathrm{~s}}$ in Fig. 6(b)] are similar to those with $\varphi_{i}=45^{\circ}$.

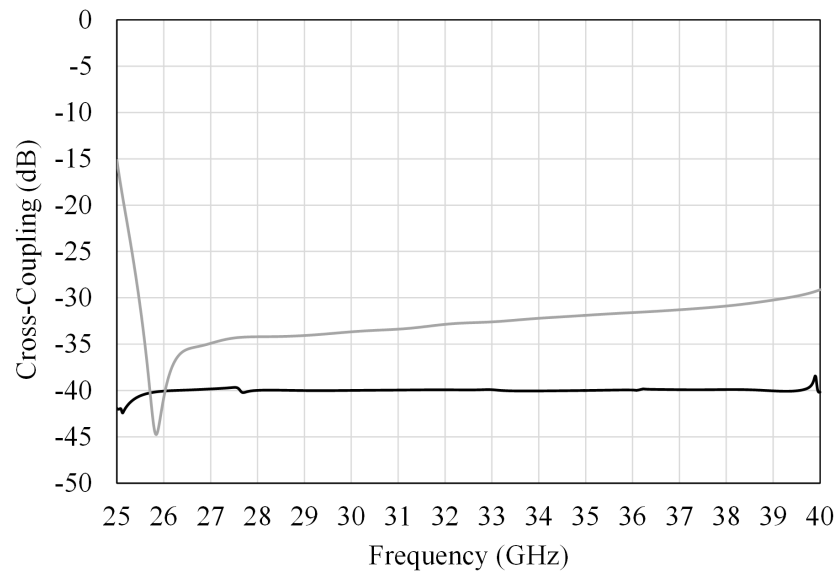

Fig. 8. Simulated cross-coupling between orthogonal modes in the designed structure: polar angle $\varphi_{i}=0^{\circ}$ [transmission between mode $\mathrm{TE}_{11 \mathrm{c}}$ in port 1 and mode $\mathrm{TE}_{11 \mathrm{~s}}$ in port 2 in Fig. 6(a)] with black solid line and polar angle $\varphi_{i}=45^{\circ}$ [transmission between mode $\mathrm{TE}_{11 \mathrm{c}}$ in port 1 and mode $\mathrm{TE}_{11 \mathrm{~s}}$ in port 2 in Fig. 6(b)] with gray solid line.

$\varphi_{i}=135^{\circ}$ positions, which are the worst-case scenarios in terms of electromagnetic performance. The results obtained from these simulations are presented in Figs. 7-9. For the insertion loss, aluminum with a typical electrical conductivity of $\sigma=3.72 \times 10^{7} \mathrm{~S} / \mathrm{m}$ has been configured for the surrounding material. Moreover, the electromagnetic simulation was used to investigate the effects of small deviations from the pure coaxial design, like the effect of a square dielectric instead of being cylindrical, and the impact of minor mechanical tolerances, like the $\pm 20-\mu \mathrm{m}$ variation in the small center pin dimensions. These simulations did not show significant differences with the results plotted in Figs. 7-9, which increase the reliability on the polar modulator performance.

From these simulation results, the good electromagnetic performance exhibited by the proposed polar modulator is evident. Port return loss is around $25 \mathrm{~dB}$ for any polar rotation angle with a degradation toward the bandwidth limits. 


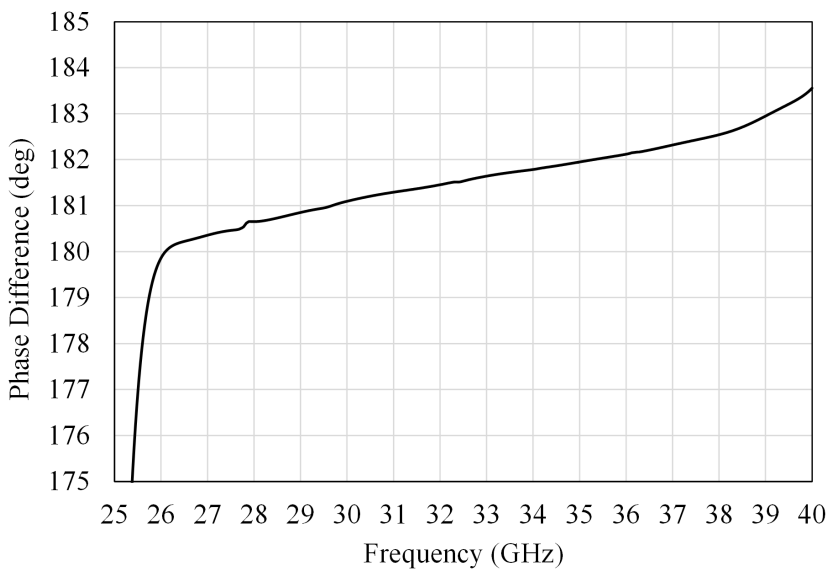

Fig. 9. Simulated phase difference between orthogonal modes in the designed polar modulator.

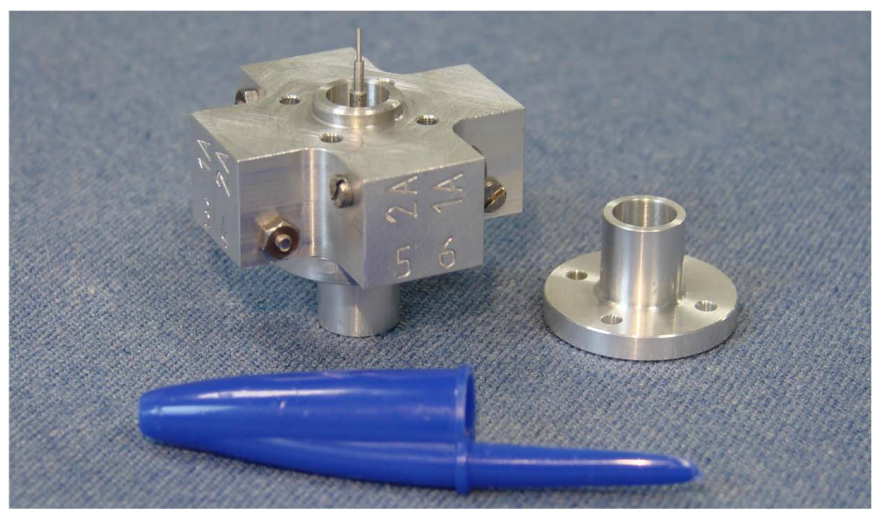

(a)

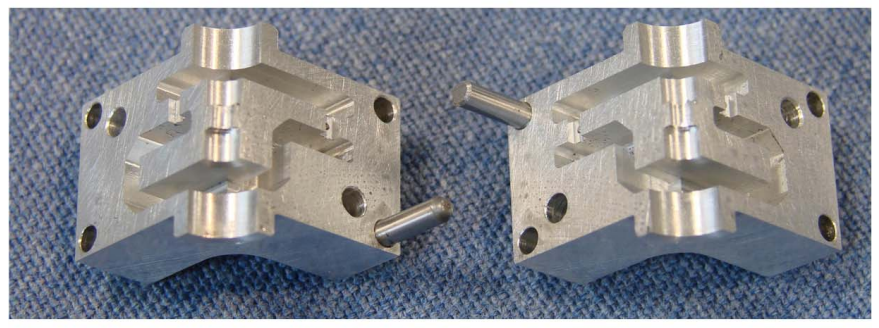

(b)

Fig. 10. (a) Manufactured polar modulator assembled. (b) Detailed view of two quadrants showing the waveguides internal routing.

These limits are defined by the cutoff frequencies of the propagated modes, where they exhibit uneven behaviors. Thus, $\mathrm{TE}_{11}$ modes in the circular waveguide have a cutoff frequency of $f_{c}=24.7 \mathrm{GHz}$, whereas the first higher order mode occurs, if excited, at $41 \mathrm{GHz}$. Therefore, the maximum bandwidth is defined by these two frequencies, being the operative bandwidth narrower than this. The performance degradation could be overcome with minor modification of the waveguides dimensions (circular and rectangular), e.g., applying the widthstep widening proposed in [20], but for this component it was decided to maintain the given dimensions in order to keep the compatibility with other subsystems in the receiver chain. The insertion losses, although they are nearly ideal,

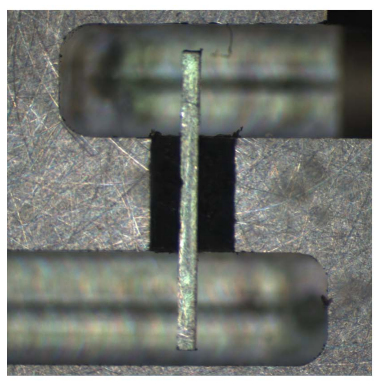

(a)

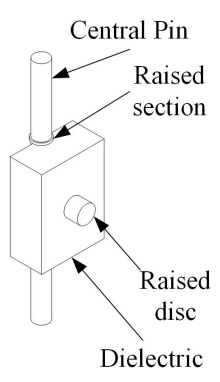

(b)

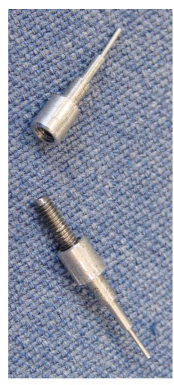

(c)
Fig. 11. (a) Close-up view of the central coaxial pin and dielectric assembled in an asymmetric waveguide-waveguide connection. (b) Sketch of the central pin and half dielectric showing the raised parts that help to keep them in place. (c) Input and output scatterers with the threaded pin used to join them.

show a flat response over the frequency range with a mean value around $0.1 \mathrm{~dB}$. Cross-coupling, which is defined as the transmission from input to output ports between orthogonal modes, is around $-40 \mathrm{~dB}$ but degraded as expected for $\varphi_{i}=45^{\circ}$, being the worst case below $-30 \mathrm{~dB}$. Finally, the phase difference between orthogonal modes is $181.5^{\circ} \pm 1.5^{\circ}$ in the whole range of interest demonstrating its suitability for the intended applications which may have phase sensitive branches. The cross-coupling is related to the phase error and differential loss between branches. For this particular application, the cross-coupling of the whole system is limited by the active gain elements which cannot be ideally matched between branches and can be no better than 15-20 dB.

\section{Polar Modulator Mechanical Design AND MANUfACTURE}

\section{A. Modulator Body}

The modulator is made from four similar blocks, which in this case, are aluminum 6061. The critical waveguide and coaxial paths have been cut into the two inner faces of each quadrant. Care is needed to symmetrically match each of the faces with its adjoining piece and to maintain a completely flat mating surface. Alignment between each piece is achieved with alignment pins and overall alignment of the assembly, input and output circular waveguide, is achieved with a tooling piece designed to hold the four quadrants together while machining the outer faces. In Fig. 10(a), the modulator assembly with one circular waveguide port removed to appreciate the scatterer is shown. A close view of two of the quadrants that form the modulator is presented in Fig. 10(b).

\section{B. Coaxial Section and Scatterer Assembly}

Fig. 11(a) shows a close-up view of the coaxial section. The square section center pin was milled in aluminum 6061 on a micromilling machine for thermal compatibility with the body. The dielectric was milled from a sheet of Rogers 5880 substrate which has graphite impregnated PTFE for its dielectric and $\varepsilon_{r}=2.2$. The center pin was machined to a tolerance of $\pm 20 \mu \mathrm{m}$ in all dimensions. No glue or solder are present in the design. Raised sections on the center pin either side of the dielectric hold it firmly in place while a raised 


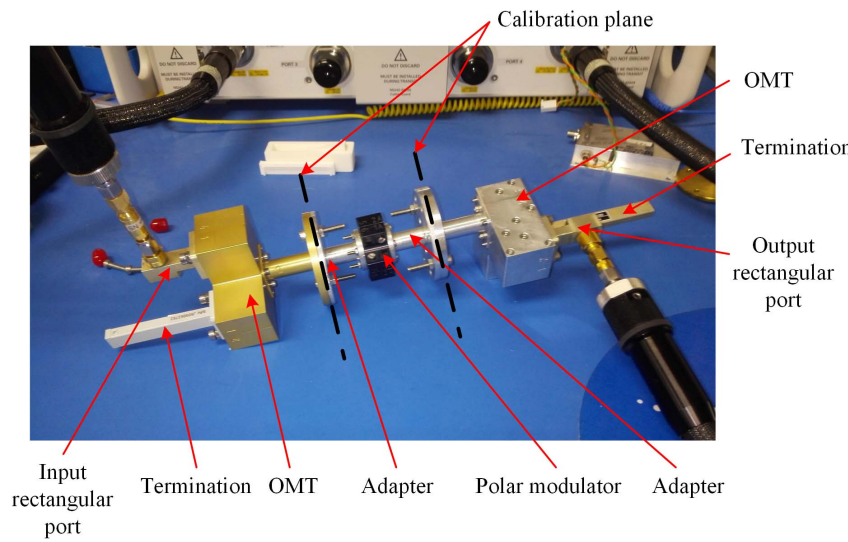

Fig. 12. Photograph of the measurement setup while taking cross-coupling data with $\varphi_{i}=0^{\circ}$. Calibration plane for all the measurements is indicated with black dashed lines.

disc in the dielectric holds it in place in the waveguide wall [see Fig. 11(b)].

Since the dielectric is built in two halves, assembly is easily carried out inserting each half into opposite faces of a modulator quadrant. The pin can be placed in one half and then the two quadrants are brought together to sandwich it between the two half dielectrics. It can be seen from Figs. 2 and 11(a) that there is no break in the outer conductor of the waveguide or coaxial section and center pin in the modulator design other than the $E$-plane split and this minimizes insertion losses.

The four-step input and output scatterers are machined individually from an aluminum piece of raw material. Once the polar modulator quadrants are held together, one scatterer with a threaded pin screwed in its base [see Fig. 11(c)] is introduced in the polarizer center cavity and conveniently machined to accommodate this element [see Fig. 10(b)] Finally, the other scatterer is screwed to the protruding threaded pin from the other circular port so that both elements pull tight together against each other and the polarizer chassis itself.

\section{Environmental Considerations}

As has been stated in the introduction to this paper, the polar modulator has been designed to spin at speed in a cryogenic environment. The modulator has been surface treated with a coating of Alodine 1200 on its conducting surfaces whereas on its outer surfaces it has been anodized for mechanical protection. The outer surface is black to aid radiative cooling. The electrical conductivity of aluminum increases when cooled to $T=10 \mathrm{~K}$ by a factor two approximately [21] causing the measured insertion loss to decrease, although not by the same factor since some of the loss is due to surface roughness and other loss is due to mismatch.

\section{Characterization and Experimental Results}

\section{A. Characterization at Room Temperature $(T=298 \mathrm{~K})$}

Measurements were taken with a VNA model E8364A from Keysight Technologies. Available OMTs [16] were used as adapters to input and output circular waveguide polarizer ports as shown in Fig. 12. The unused rectangular ports

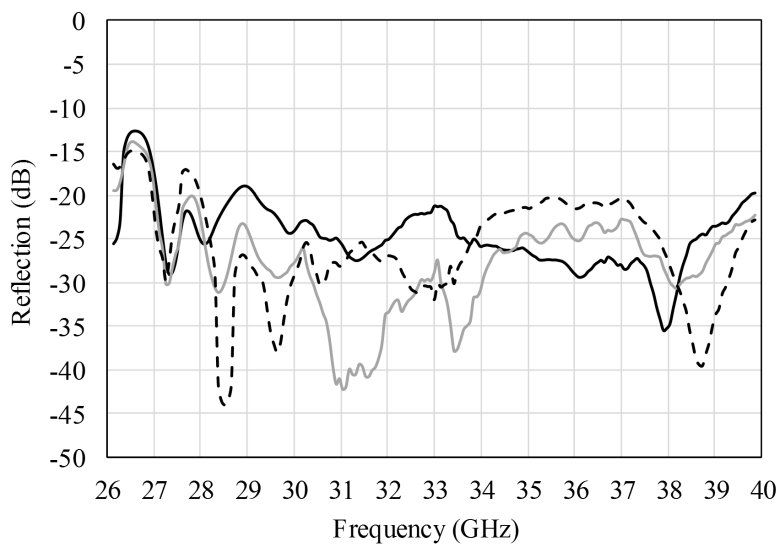

Fig. 13. Measured return loss for the proposed polar modulator: polar angle $\varphi_{i}=0^{\circ}$ with black solid line, polar angle $\varphi_{i}=90^{\circ}$ with black dashed line, and polar angle $\phi_{i}=45^{\circ}$ with gray solid line.

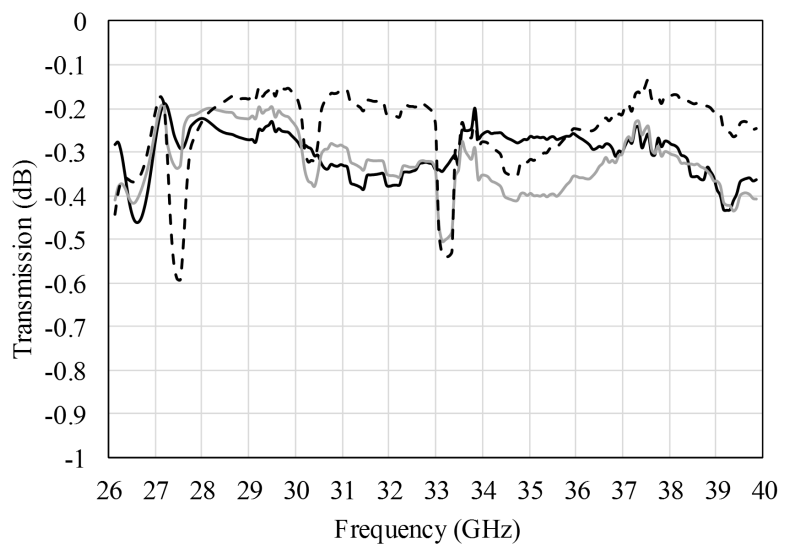

Fig. 14. Measured insertion loss for the proposed polar modulator: polar angle $\varphi_{i}=0^{\circ}$ with black solid line, polar angle $\varphi_{i}=90^{\circ}$ with black dashed line, and polar angle $\varphi_{i}=45^{\circ}$ with black gray line.

of these OMTs were loaded with a commercial rectangular waveguide termination. In addition, adapters with eight alignment holes equi-spaced (every $45^{\circ}$ ) about the waveguide axis allowed rotation of the polar modulator with respect to the OMT, obtaining the necessary angles to perform all the measurements, i.e., $\varphi_{i}=0^{\circ}, 45^{\circ}$, and $90^{\circ}$, etc. A thrureflect-line calibration was carried out with the OMT circular port as the reference plane (see Fig. 12) using purpose-built calibration shorts and a $\lambda_{g} / 4$ delay line of 3-mm length and 10-ps delay, being $\lambda_{g}$ the waveguide wavelength at center frequency $(33 \mathrm{GHz})$. Both the calibration and measurement have been performed multiple times, improving the measurement technique for each repetition. The concentricity and polar alignment have been found to be critical in making a good measurement.

Return loss and insertion loss results for $\varphi_{i}=0^{\circ}$ are obtained with the configuration shown in Fig. 12, but with input and output rectangular ports following the same direction. On the other hand, the same measurements but for $\varphi_{i}=90^{\circ}$ are recorded by changing these rectangular accesses to the orthogonal ports in each OMT. These results are shown in Fig. 13 for return loss and Fig. 14 for insertion loss. In the 


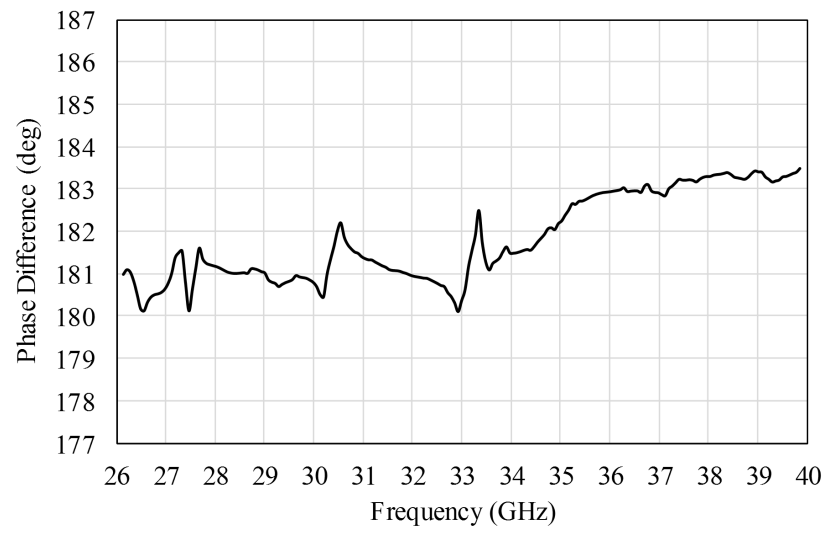

Fig. 15. Measured phase difference between the modes propagated through the two different branches in the polar modulator.

case of $\varphi_{i}=45^{\circ}$ and $135^{\circ}$, the polar modulator is rotated $45^{\circ}$ with respect to the OMT axis and return loss and insertion loss are obtained when the measurement rectangular ports are in orthogonal configuration, i.e., the input and output ports have perpendicular directions. These last results are also included in Figs. 13 and 14. Results for $\varphi_{i}=135^{\circ}$ are omitted because they are similar to those of $\varphi_{i}=45^{\circ}$.

From these plots, a return loss better than $20 \mathrm{~dB}$ can be inferred for all the possible polar angles in the whole frequency range. Regarding the insertion loss, a mean value around $0.3 \mathrm{~dB}$ is obtained for all rotation positions. This value is higher than predicted in Fig. 7 but the simulations are based on ideal electrical conductivities and did not take into consideration the material roughness and adapter loss, which has an impact at these frequencies.

Using the transmission measurements at $\varphi_{i}=0^{\circ}$ and $90^{\circ}$, the phase difference between the two propagated modes can be calculated. This difference is plotted in Fig. 15 where a mean value of $181.8^{\circ} \pm 1.7^{\circ}$ can be appreciated. This result agrees with the values obtained through the simulations and presented in Fig. 9.

Both in Figs. 14 and 15 some spikes in the plots are present at certain frequencies. These rapid variations are attributed to the alignment of the polar modulator within the measurement setup and also the alignment of the scatterers in the turnstile junctions. From our experience, and as stated previously, errors in the positioning of these pieces and the modulator itself have a significant impact on the quality of the measured results. In spite of this and due to the broadband feature of the intended application, these uneven values at some single frequencies do not spoil the overall performance.

Finally, cross-coupling for $\varphi_{i}=0^{\circ}$ is measured with the configuration in Fig. 12. In the case of $\varphi_{i}=45^{\circ}$, the polar modulator is rotated $45^{\circ}$ and the cross-coupling is the transmission when both rectangular ports are parallel. Cross-coupling for $\varphi_{i}=90^{\circ}$ and $\varphi_{i}=135^{\circ}$ are redundant. Results obtained in these last two measurements are presented in Fig. 16, where typical values better than $-35 \mathrm{~dB}$ are demonstrated with a worst-case value better than $-30 \mathrm{~dB}$ for $\varphi_{i}=45^{\circ}$. These results are similar to those predicted by simulations and plotted

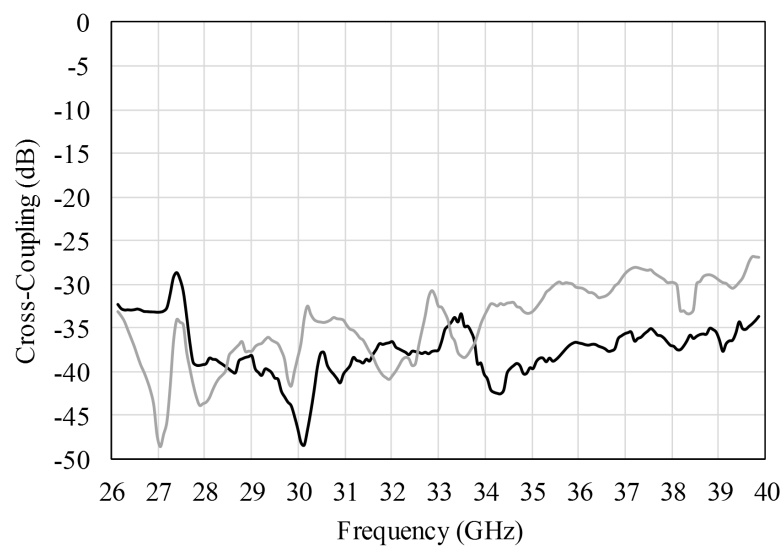

Fig. 16. Measured cross-coupling between orthogonal modes with the polar modulator at $\varphi_{i}=0^{\circ}$ (black line) and $\varphi_{i}=45^{\circ}$ (gray line).

TABLE I

Results Comparison Between the Published HWPs And WPMs

\begin{tabular}{|c|c|c|c|c|c|c|c|}
\hline Ref. & Technol. & $\begin{array}{l}\text { BW } \\
(\%)\end{array}$ & $\begin{array}{c}\text { Return } \\
\text { Loss } \\
\text { (dB) }\end{array}$ & $\begin{array}{c}\text { Insertion } \\
\text { Loss } \\
\text { (dB) }\end{array}$ & $\begin{array}{c}\text { Cross- } \\
\text { Coupling } \\
\text { (dB) }\end{array}$ & $\begin{array}{c}\text { Phase } \\
\text { Shif (deg) }\end{array}$ & Complexity \\
\hline [14] & WPM & $\begin{array}{c}29 \\
(82-110) \\
\end{array}$ & 38 & 0.27 & -36.5 & $180.4 \pm 0.7$ & Medium \\
\hline$[5]$ & WPM & $\begin{array}{c}28 \\
(83-110) \\
\end{array}$ & $>15.2$ & 0.97 & N/A & N/A & High \\
\hline \multirow{2}{*}[10]{} & \multirow{2}{*}{ HWP } & $\begin{array}{c}30 \\
(85-115)\end{array}$ & $17.2^{*}$ & $0.2 * *$ & $<-28$ & $183 \pm 1.5$ & High \\
\hline & & $\begin{array}{c}74 \\
(85-185) \\
\end{array}$ & $15.4^{*}$ & $0.31 * *$ & $<-29$ & $180.1 \pm 3.2$ & High \\
\hline \multirow{2}{*}{ [11] } & \multirow{2}{*}{ HWP } & $\begin{array}{c}30 \\
(127-173)\end{array}$ & N/A & $0.22 * *$ & -25.5 & $183.2 \pm 1.7$ & High \\
\hline & & $\begin{array}{c}40 \\
(120-180) \\
\end{array}$ & N/A & $0.2 * *$ & -25 & $182.5 \pm 2.2$ & High \\
\hline [12] & HWP & $\begin{array}{c}25 \\
(78-100) \\
\end{array}$ & N/A & $0.46^{* *}$ & -28 & $180.4 \pm 2.9$ & High \\
\hline $\begin{array}{l}\text { This } \\
\text { work }\end{array}$ & WPM & $\begin{array}{c}40 \\
(26.5-40) \\
\end{array}$ & 28 & 0.3 & -34 & $181.8 \pm 1.7$ & Low \\
\hline
\end{tabular}

Values obtained as mean values across the stated bandwidth for the worst-case scenario.

* Value calculated as RL $(\mathrm{dB})=-10 \cdot \log$ (Reflection coefficient)

** Value calculated as IL $(\mathrm{dB})=-10 \cdot \log$ (Transmission coefficient)

in Fig. 8 taking into account the difficulty in making this measurement.

All these reported results may be affected by other subsystems in the receiver, especially the antenna feed horn placed just before the polar modulator. Simulations have demonstrated how a poor antenna return loss may have an impact on the polar modulator isolation, defined as the transmission between orthogonal modes at the output port when the input port is connected to the feed horn. Moreover, this impact is dependent upon the polar angle, being the worst isolation value for $\varphi_{i}=45^{\circ}$ and $\varphi_{i}=135^{\circ}$. These simulations have shown that, for an antenna feed horn return loss better than $20 \mathrm{~dB}$, the influence of this component on the polar modulator performance is practically negligible, and therefore these horns are designed with a return loss of $25 \mathrm{~dB}$ as mean values across the band in the QUIJOTE project.

Table I shows a comparison between the proposed structure and other HWPs and WPMs present in the literature. As can be seen, the presented polar modulator is a good alternative for polarimetry in radio astronomy, and in some cases, may be advantageous. 


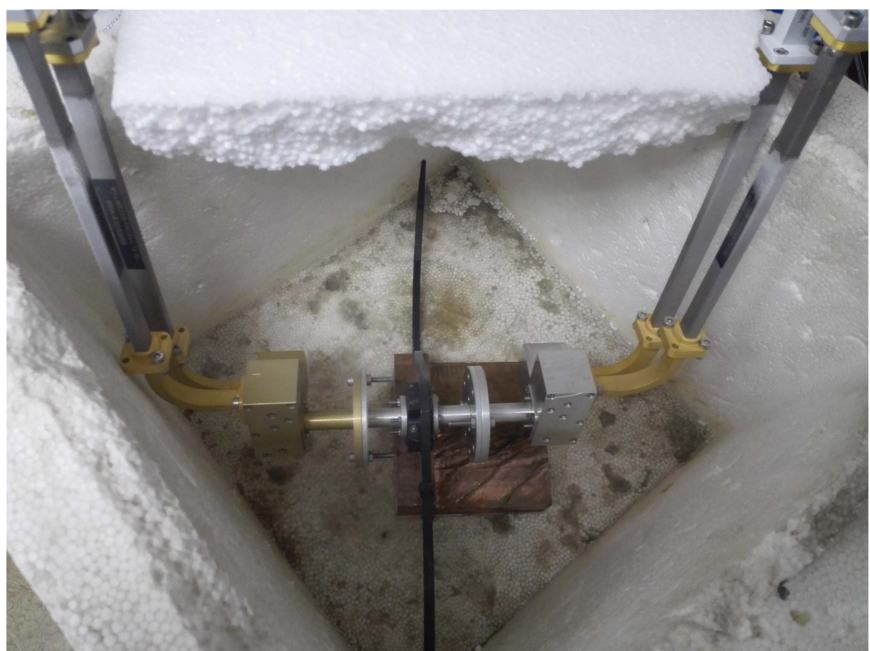

Fig. 17. Measurement setup at cryogenic temperature.

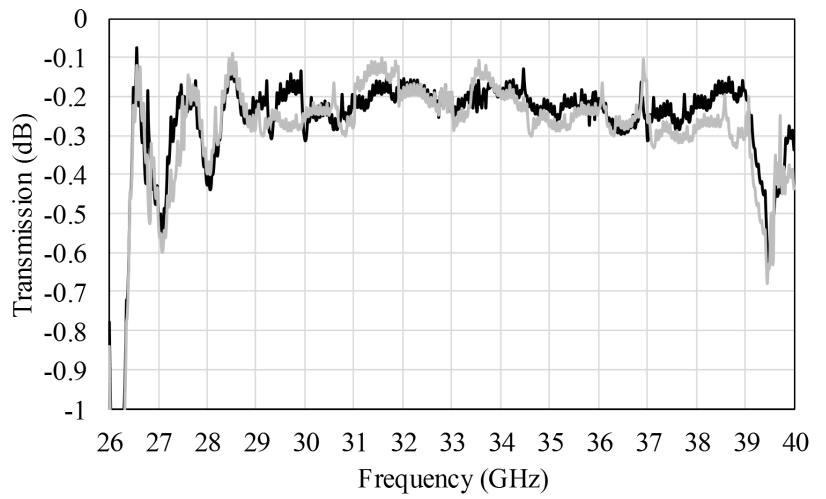

Fig. 18. Measured insertion loss at cryogenic temperature for $\varphi_{i}=0^{\circ}$ (black line) and for $\varphi_{i}=90^{\circ}$ (gray line).

\section{B. Characterization at Cryogenic Temperature ( $T=80 \mathrm{~K})$}

Due to the simplicity of the design, the change in mechanical dimensions of aluminum 6061 and dielectric parts are considered not to be enough to cause a significant effect on the electromagnetic properties of this wideband component. Only differential changes can cause significant changes in the measurements and the modulator is designed to minimize them. However, some cryogenic tests have been performed in order to check the performance variations with the temperature. The lack of a suitable cryostat for carrying out these tests to the stand-alone polar modulator at temperatures down to $10-20 \mathrm{~K}$ has imposed a limited measurement setup using liquid nitrogen $(T=77 \mathrm{~K})$.

This setup is shown in Fig. 17. The system is calibrated at room temperature with the same reference plane as used in the room temperature measurements already described, and then, the polar modulator is connected between the OMTs and tied to a copper block which is immersed in liquid nitrogen. This procedure provides limited measurement accuracy since the waveguides and the OMTs have performance variations with temperature, but curve trends can be obtained from the results. Furthermore, the S-parameters were measured with the

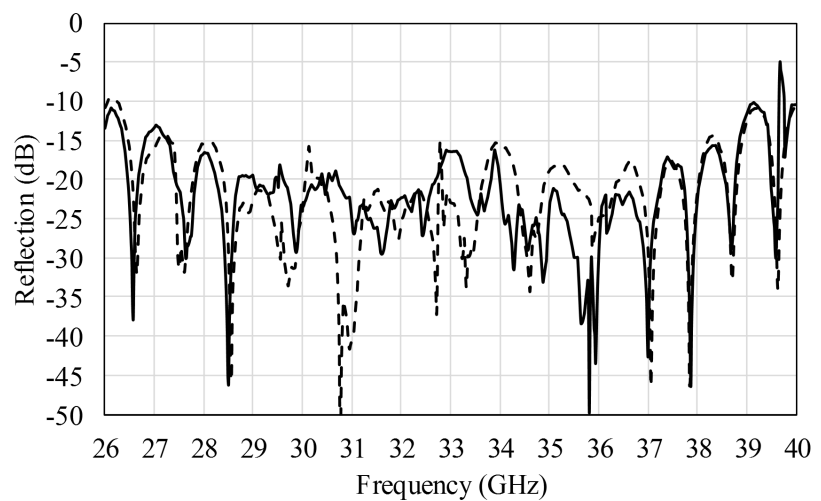

Fig. 19. Measured reflection at cryogenic temperature for $\varphi_{i}=0^{\circ}$ (solid line) and $\varphi_{i}=90^{\circ}$ (dashed line).

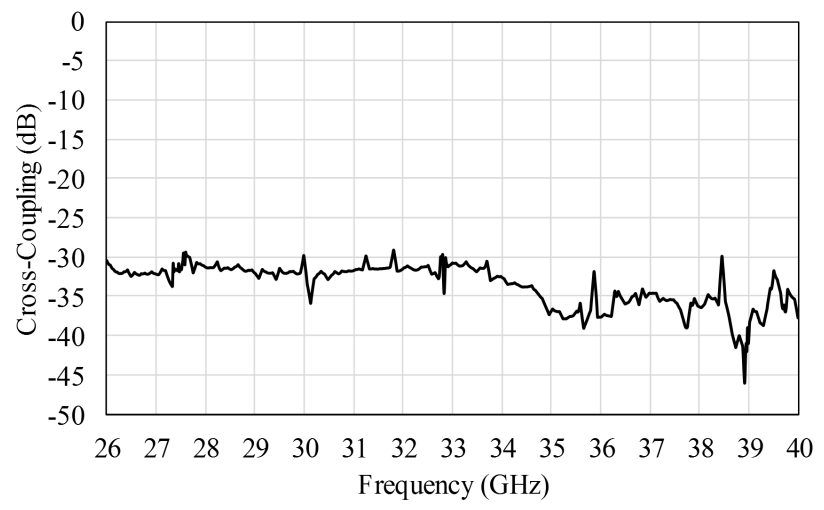

Fig. 20. Measured cross-coupling at cryogenic temperature for $\varphi_{i}=0^{\circ}$.

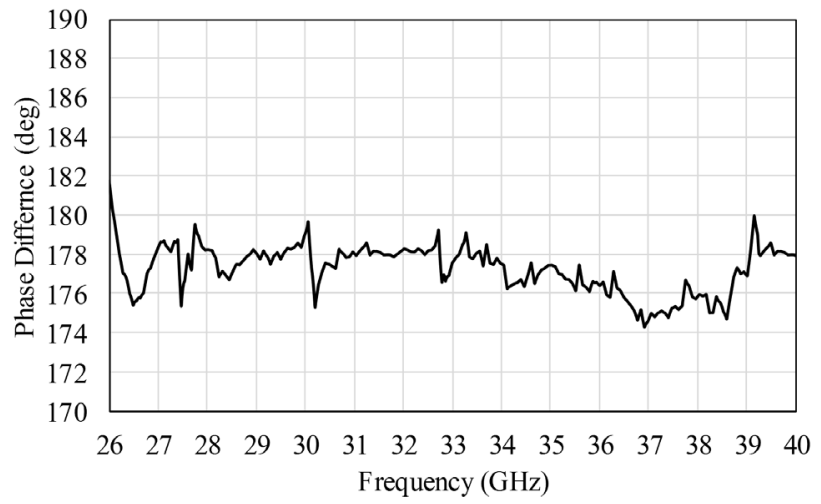

Fig. 21. Measured phase difference between propagated modes at cryogenic temperature.

four VNA ports connected. The E8364A allows simultaneous three-port and two-port calibration and measurements through any combination of the ports. This was essential to provide consistency between the measurements made when the polar modulator was cooled. In general, the measured insertion loss, plotted in Fig. 18, is reduced from $0.3 \mathrm{~dB}$ at room temperature to around $0.2 \mathrm{~dB}$ at $T=80 \mathrm{~K}$ (approximately), which represents around a 30\% variation. The transmission degradation toward band edges is believed to be related with the setup performance variation upon cooling. The test setup was previously cooled without the polar modulator (and with 
the two OMTs connected directly) and showed a similar degradation. This degradation also affects the reflection results shown in Fig. 19. However, most of the frequency band presents return loss values around $20 \mathrm{~dB}$, close to the curves obtained at room temperature.

The cross-coupling is measured with the polar angle at $\varphi_{i}=0^{\circ}$ and the obtained result is presented in Fig. 20. A mean value below $-30 \mathrm{~dB}$ can be appreciated, which is similar to the corresponding curve at room temperature shown in Fig. 16. Finally, the phase difference between propagated modes remains stable as expected. The measured curve is plotted in Fig. 21 obtaining a mean value around $178^{\circ}$ even with the aforementioned measurement difficulties. The different spikes present in all the results are mainly due to misalignment issues in the measurement setup.

\section{CONCLUSION}

A full-band (40\% relative bandwidth) polar modulator designed completely in waveguide technology, following a compact and scalable configuration, and intended for polarimetry applications in radio astronomy has been presented. The shift structure relies on symmetric-asymmetric waveguidecoaxial-waveguide field couplings that provide wideband $180^{\circ}$ phase difference in nature. The polar modulator is completed with input and output turnstile junctions that offer good isolation between branches. Mechanically, the polarimeter is divided into four quarters that are easily machined and assembled. This compact design can be cryogenically cooled and spun at high speed which makes it very suitable for receivers suffering from significant $1 / f$ noise.

The proposed design has been demonstrated with a Kaband $(26-40 \mathrm{GHz})$ polar modulator showing $-25,-0.3$, and $-37.5 \mathrm{~dB}$ of return loss, insertion loss, and cross-coupling, respectively, as mean values across the band for a polar angle of $\varphi_{i}=0^{\circ}$. Results for other polar angles are very similar including the theoretical worst case of $\varphi_{i}=45^{\circ}$. Finally, the measured phase difference between propagated modes is $181.8^{\circ} \pm 1.7^{\circ}$.

When cooled to $T=80 \mathrm{~K}$ approximately, the polar modulator exhibits a stable performance. While the insertion loss is reduced by about $30 \%$ as expected from the increased electrical conductivity of aluminum, the cross-coupling and phase difference present similar values to those obtained at room temperature.

\section{REFERENCES}

[1] R. P. Torres and M. F. Catedra, "Analysis and design of a two-octave polarization rotator for microwave frequency," IEEE Trans. Microw. Antennas Propag., vol. 41, no. 2, pp. 208-213, Feb. 1993.

[2] R. Lech, M. Mazur, and J. Mazur, "Analysis and design of a polarizer rotator system," IEEE Trans. Microw. Antennas Propag., vol. 56, no. 3, pp. 844-847, Mar. 2008.

[3] T.-K. Wu, "Meander-line polarizer for arbitrary rotation of linear polarization," IEEE Microw. Guided Wave Lett., vol. 4, no. 6, pp. 199-201, Jun. 1994.

[4] M. H. Abitbol. (Jul. 2017). "CMB-S4 technology book, first edition." [Online]. Available: https://arxiv.org/abs/1706.02464v2

[5] P. A. R. Ade et al., "Polarization modulators for CMBPol," J. Phys., Conf. Ser., vol. 155, no. 1, p. 012006, 2009.

[6] D. T. Chuss et al., "Properties of a variable-delay polarization modulator," Appl. Opt., vol. 51, no. 2, pp. 197-208, 2012.
[7] D. T. Chuss, E. J. Wollack, G. Pisano, S. Ackiss, K. U-Yen, and M. W. Ng, "A translational polarization rotator," Appl. Opt., vol. 51, no. 28 , pp. 6824-6830, 2012.

[8] J. Zhang, P. A. R. Ade, P. Mauskopf, L. Moncelsi, G. Savini, and N. Whitehouse, "New artificial dielectric metamaterial and its application as a terahertz antireflection coating," Appl. Opt., vol. 48, no. 35 , pp. 6635-6642, Dec. 2009.

[9] G. Pisano, "Systematic polarisation effects introduced by lenses and by axial birefringent half-wave plates used in CMB experiments in the millimeter region," Proc. SPIE, vol. 4843, pp. 348-359, Feb. 2003.

[10] G. Pisano, G. Savini, P. A. R. Ade, V. Haynes, and W. K. Gear, "Achromatic half-wave plate for submillimeter instruments in cosmic microwave background astronomy: Experimental characterization," Appl. Opt., vol. 45, no. 27, pp. 6982-6989, Sep. 2006.

[11] G. Pisano, G. Savini, P. A. R. Ade, and V. Haynes, "Metal-mesh achromatic half-wave plate for use at submillimeter wavelengths," Appl. Opt., vol. 47, no. 33, pp. 6251-6256, Nov. 2008.

[12] G. Pisano, M. W. Ng, V. Haynes, and B. Maffei, "A broadband metalmesh half-wave plate for millimetre wave linear polarisation rotation," Prog. Electromagn. Res. M, vol. 25, pp. 101-114, Jul. 2012.

[13] G. Pissano et al., "Development of large radii half-wave plates for CMB satellite missions," Proc. SPIE, vol. 9153, p. 915317, Jul. 2014.

[14] G. Pisano, S. Melhuish, G. Savani, L. Piccirillo, and B. Maffei, "A broadband W-band polarization rotator with very low cross polarization," IEEE Microw. Wireless Comp. Lett., vol. 21, no. 3, pp. 127-129, Mar. 2011.

[15] J. A. Rubino-Martin et al., "The QUIJOTE CMB experiment," in Proc. 8th Sci. Meeting Spanish Astron. Soc., Santander, Spain, Jul. 2008, pp. 1-9. [Online]. Available: https://arxiv.org/abs/0810.3141

[16] J. L. Cano, A. Tribak, R. Hoyland, A. Mediavilla, and E. Artal, "Full band waveguide turnstile junction orthomode transducer with phase matched outputs," Int. J. RF Microw. Comput.-Aided Eng., vol. 20, no. 3, pp. 333-341, May 2010.

[17] R. Génova-Santos et al., "QUIJOTE scientific results-I. Measurements of the intensity and polarisation of the anomalous microwave emission in the Perseus molecular complex," Monthly Notices Roy. Astronom. Soc., vol. 452, no. 4, pp. 4169-4182, Aug. 2015.

[18] F. Patat and M. Romaniello, "Error analysis for dual-beam optical linear polarimetry," Publications Astronom. Soc. Pacific, vol. 118, no. 839, pp. 146-161, Jan. 2006.

[19] R. J. Hoyland et al., "The status of the QUIJOTE multi-frequency instrument," Proc. SPIE, vol. 8452, p. 845233, Oct. 2012.

[20] A. Tribak, J. L. Cano, A. Mediavilla, and M. Boussouis, "Octave bandwidth compact turnstile-based orthomode transducer," IEEE Microw. Wireless Compon. Lett., vol. 20, no. 10, pp. 539-541, Oct. 2010.

[21] S. W. Van Sciver, Helium Cryogenics, 2nd ed. New York, NY, USA: Springer, 2012, ch. 2, pp. 29-33.

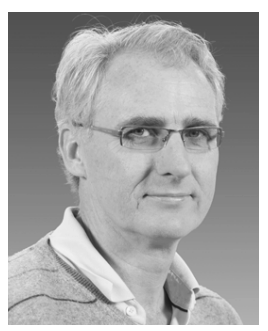

Roger Hoyland received the degree in applied physics from the University of Bath, Bath, U.K., in 1988, and the master's degree in intrumentational cosmology from the Victoria University of Manchester, Manchester, U.K.

$\mathrm{He}$ was with the Izaña Observatory, Tenerife, Spain, where he was involved in the cooled microwave receivers at 10,15 , and $33 \mathrm{GHz}$. $\mathrm{He}$ joined the PLANCK Surveyor Project in 1998 and specialized in the design of a cooled phase switch suitable for the LFI radiometers. He is currently supervising a Ph.D. student at the Instituto de Astrofísica de Canarias, Santa Cruz de Tenerife, Spain, within the QUIJOTE project, where he also holds a permanent position.

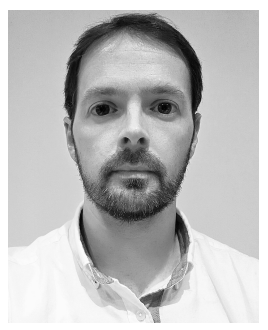

Juan L. Cano was born in Torrelavega, Spain, in 1979. He received the telecommunications engineering and $\mathrm{Ph} . \mathrm{D}$. degrees from the University of Cantabria, Santander, Spain, in 2004 and 2010, respectively.

$\mathrm{He}$ is currently a Researcher with the University of Cantabria, where he collaborates in the development and measurement of microwave receivers for radio astronomy and satellite applications. His current research interests include the design and testing of low-noise amplifiers in MIC and MMIC technologies both at room and cryogenic temperatures, the design of different hardware for feed networks, and the development of new technologies for efficient subsystems in multipixel microwave cameras. 


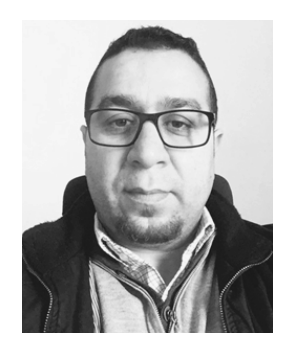

Abdelwahed Tribak was born in Larache, Morocco, in 1981. He received the M.Sc. degree in physics from Abdelmalek Essaadi University, Tétouan, Morocco, in 2006, and the master's degree in communications engineering and Ph.D. degree in telecommunications from the University of Cantabria, Santander, Spain, in 2008 and 2011, respectively.

From 2006 to 2011, he was with the Department of Communications Engineering, University of Cantabria. Since 2011, he has been a Professor with the National Institute of Poste and Telecommunication, Rabat, Morocco. His current research interests include microwave circuits and systems and antenna feed subsystems for satellite and radio astronomy applications.

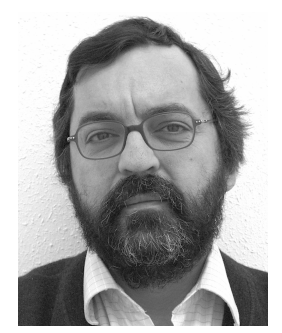

Angel Mediavilla was born in Santander, Spain, in 1955. He received the degree and Doctor of Physics (Electronic) degree (Hons.) from the University of Cantabria, Santander, in 1978 and 1983, respectively.

From 1980 to 1983, he was an Ingenieur Stagiaire with THOMSON-CSF. He possesses wide experience in the analysis and optimization of nonlinear microwave active devices. His current research interests include nonlinear MESFET/HEMT and HBT device modeling with special application to the large-signal computer design and new waveguide structures for antenna feed systems.

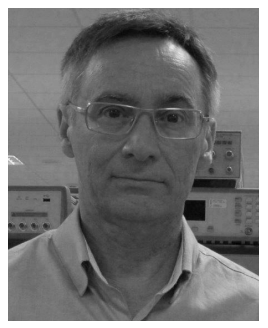

Eduardo Artal (M'81) received the telecommunications engineering and Ph.D. degrees from the Technical University of Catalonia, Barcelona, Spain, in 1976 and 1982, respectively.

From 1976 to 1990, he was an Assistant Professor with the Technical University of Catalonia. From 1979 to 1981, he was with the Mier Allende S.A. Company, Barcelona, where he was involved in TV and FM radio reemitter development. Since 1990, he has been a Professor with the University of Cantabria, Santander. His current research interests include microwave circuits and systems and low-noise millimeter-wave amplifiers and receivers. 